Pacific Journal of Mathematics

WCAL AEES FoR PRoFNITIE GRO 


\title{
LOCAL $\Lambda$ SETS FOR PROFINITE GROUPS
}

\author{
M. F. HUTCHINSON
}

Let $E$ be a subset of the dual $\hat{G}$ of a profinite group $G$. It is shown that if $E$ is a local $A$ set then the degrees of the elements of $E$ must be bounded. It follows that $\hat{G}$ contains an infinite Sidon set if and only if $\hat{G}$ has infinitely many elements of the same degree. This characterisation is the same as one previously obtained for compact Lie groups.

Preliminaries. Let $G$ be a compact group with normalized Haar measure $\lambda_{G}$. For $p \in[1, \infty[$ the Banach space of $p$ th power integrable complex-valued functions on $G$ is denoted $\left(L^{p}(G),\|\cdot\|_{p}\right)$. The dual object $\hat{G}$ of $G$ is taken to be a maximal set of pairwise inequivalent continuous irreducible unitary representations of $G$. For each $\sigma \in \hat{G}$ let $d_{o}$ be the degree or dimension of the representation space of $\sigma$ and let $\chi_{\sigma}$ denote its trace. The Fourier transform of $f \in L^{1}(G)$ is the matrix-valued function $\hat{f}$ on $\hat{G}$ defined by

$$
\hat{f}(\sigma)=\int_{G} f(x) \sigma\left(x^{-1}\right) d \lambda_{G}(x) \quad(\sigma \in \hat{G}) .
$$

If $E$ is a subset of $\hat{G}$ let $S_{E}(G)$ denote the set of all trigonometric polynomials on $G$ whose Fourier transforms are supported by just one element of $E$. For $p \in] 1, \infty$ [ call $E$ a local $\Lambda_{p}$ set if there exists a positive constant $\kappa$ such that

$$
\|f\|_{p} \leqq \kappa\|f\|_{1}
$$

for all $f \in S_{E}(G)$. Call $E$ a local central $\Lambda_{p}$ set if there exists a positive constant $\kappa$ such that

$$
\left\|\chi_{o}\right\|_{p} \leqq \kappa\left\|\chi_{o}\right\|_{1}
$$

for all $\sigma \in E$. Further, $E$ is a local $\Lambda$ set if there exists a positive constant $\kappa$ such that

$$
\|f\|_{p} \leqq \kappa p^{1 / 2}\|f\|_{2}
$$

for all $f \in S_{E}(G)$ and all $\left.p \in\right] 2, \infty\left[\right.$. A local $\Lambda$ set is local $A_{p}$ for every $p \in] 1, \infty[$. See $\S 37$ of [4] for a general introduction to the theory of lacunary sets.

If $G$ is profinite and $\left\{N_{\alpha}\right\}_{\alpha \in A}$ is a neighborhood base at the identity consisting of open normal subgroups of $G$ then each $\sigma \in \widehat{G}$ has kernel containing some $N_{\alpha}$ by Lemma (28.17) of [4]. Thus we 
may write

$$
\widehat{G}=\bigcup_{\alpha \in A}\left(G / N_{\alpha}\right)^{\wedge}
$$

if we identify a representation of a quotient of $G$ with a representation of $G$. We say $G$ is tall if for each positive integer $n$ there are only finitely many elements of $\hat{G}$ of degree $n$. Structural characterisations of tall profinite groups are given in [7]. We will show that a profinite group $G$ admits an infinite (local) Sidon set if and only if $G$ is not tall.

The main theorem.

LEMma 1. Let $H$ be an open subgroup of a compact group $G$ having index $[G: H]=t$ and let $\left\{x_{1}=1, x_{2}, \cdots, x_{t}\right\}$ be a set of left coset representatives for $H$. Then we have

$$
\int_{G} f(x) d \lambda_{G}(x)=t^{-1} \sum_{i=1}^{t} \int_{H} f\left(x_{i} h\right) d \lambda_{H}(h)
$$

for every continuous complex-valued function $f$ on $G$.

Proof. It is easily verified that the right hand side of (1) defines a positive left invariant normalized measure on $G$.

Lemma 2. Let $G$ and $H$ be as in Lemma 1. If $\sigma \in \hat{G}$ and $\left|\chi_{o}(h)\right|=d_{o}$ for all $h \in H$ then

$$
\left\|\chi_{\sigma}\right\|_{p} \geqq d_{\sigma} / t^{1^{\prime} p}
$$

for all $p \in[1, \infty[$.

Proof. By Lemma 1 we have

$$
\begin{aligned}
\left\|\chi_{\sigma}\right\|_{p}^{p} & =t^{-1} \sum_{i=1}^{t} \int_{H}\left|\chi_{\sigma}\left(x_{i} h\right)\right|^{p} d \lambda_{H}(h) \\
& \geqq t^{-1} \int_{H}\left|\chi_{\sigma}(h)\right|^{p} d \lambda_{H}(h) \\
& =t^{-1} d_{\sigma}^{p}
\end{aligned}
$$

from which the lemma follows at once.

Lemma 3. Let $G$ and $H$ be as in Lemma 1 and let $f$ be a continuous complex-valued function on $G$ which vanishes outside $H$. Define a continuous function $g$ on $H$ by setting $g(h)=f(h)$ for all $h \in H$. Then for $p \in[1, \infty[$ we have

$$
\|f\|_{p}=t^{-1 / p}\|g\|_{p}
$$


Proof. This follows immediately from Lemma 1.

Lemma 4. Let $G$ be a compact group and let $E \subset \hat{G}$ be a $\Lambda_{p}$ set for some $p \in] 1, \infty[$. Suppose that for each $\sigma \in E$ there is an open subgroup $H_{\sigma}$ of $G$ of index $t_{\sigma}$ and a representation $\tau \in \hat{H}_{\sigma}$ such that $\sigma$ is equivalent to the induced representation $\tau^{G}$. Then we have

$$
\sup \left\{t_{\sigma}: \sigma \in E\right\}<\infty \text {. }
$$

Proof. For each $\sigma \in E$ define a continuous function $f_{\sigma}$ on $G$ by setting

$$
f_{\sigma}(x)=\left\{\begin{array}{lll}
\chi_{-}(x) & \text { for } & x \in H_{\sigma} \\
0 & \text { for } & x \in G-H_{\sigma}
\end{array}\right.
$$

Now for each $\rho \in \hat{G}$ we have

$$
\left.\rho\right|_{H_{\sigma}} \cong \bigoplus_{v \in \hat{H}_{\sigma}} n_{\rho}(\nu) \cdot \nu
$$

where $n_{\rho}(\nu)$ denotes the multiplicity of $v$ in the representation of $H_{\sigma}$ obtained by restricting the domain of $\rho$. Since we have

$$
\hat{f}_{\sigma}(\rho)=t_{\sigma}^{-1} \int_{H_{\sigma}} \chi_{\tau}(h) \rho\left(h^{-1}\right) d \lambda_{H_{\sigma}}(h)
$$

by Lemma 1, the orthogonality relations for $H_{\sigma}$ then show that $\hat{f}_{o}(\rho)$ vanishes for all $\rho \in \hat{G}$ for which $n_{\rho}(\tau)=0$. By Frobenius reciprocity, these are all $\rho$ except $\sigma \cong \tau^{G}$ and so we have that $f_{\sigma} \in S_{E}(G)$. Using Lemma 3 and a standard inequality for $L^{p}$ spaces (see (13.17) of [5]) we have

$$
\begin{aligned}
\left\|f_{\sigma}\right\|_{p} & =t_{\sigma}^{-1^{\prime} p}\left\|\chi_{\tau}\right\|_{p} \\
& \geqq t_{\sigma}^{-1 / p}\left\|\chi_{\tau}\right\|_{1} \\
& =t_{\sigma}^{1-1 / p}\left\|f_{\sigma}\right\|_{1} .
\end{aligned}
$$

Now if $E$ is a local $\Lambda_{p}$ set then there is a positive constant $\kappa$ such that

$$
\left\|f_{\sigma}\right\|_{p} \leqq \kappa\left\|f_{\sigma}\right\|_{1} \text { for all } \sigma \in E
$$

so the above calculation shows that

$$
t_{\sigma}^{1-1 / p} \leqq \kappa \text { for all } \sigma \in E
$$

and this can only happen if

$$
\sup \left\{t_{o}: \sigma \in E\right\}<\infty .
$$

Lemma 5. (Jordan, Blichfeldt). Let $G$ be a finite complex linear 
group of degree $n$. Then $G$ has an abelian normal subgroup $A$ such that

$$
[G: A]<6^{4 n^{2} / \log n} \text {. }
$$

Proof. See p. 177 of [3] and observe that

$$
n ! 6^{\pi(n+1)+1}<6^{4 n^{2} / \log n}
$$

where $\pi(m)$ denotes the number of primes not exceeding $m$.

THEOREM. Let $G$ be a profinite group and let $E \subset \hat{G}$ be a local $\Lambda$ set. Then we have

$$
\sup \left\{d_{\sigma}: \sigma \in E\right\}<\infty \text {. }
$$

Proof. For each $\sigma \in E$ we may apply Lemma 5 to the finite group $G / \operatorname{ker} \sigma$ to obtain an open normal subgroup $A_{\sigma}$ of $G$ such that $A_{\sigma} \supset \operatorname{ker} \sigma, A_{\sigma} / \operatorname{ker} \sigma$ is abelian and

$$
\left[G: A_{\sigma}\right]<6^{4 d_{\sigma}^{2} / \log d_{\sigma}} .
$$

By Clifford's theorem (see $\S 14$ of [3]), for each $\sigma$ there is an irreducible 1-dimensional representation $\xi_{\sigma}$ of $A_{\sigma}$ and positive integers $e_{\sigma}$ and $t_{\sigma}$ such that

$$
\left.\sigma\right|_{\Delta_{\sigma}} \cong e_{\sigma} \cdot\left\{\xi_{\sigma}^{x_{1}} \oplus \cdots \oplus \xi_{\sigma}^{x_{\sigma}}\right\}
$$

where $\left\{x_{1}=1, x_{2}, \cdots, x_{t_{o}}\right\}$ is a set of left coset representatives of the inertia group $S_{\sigma}$ given by

$$
S_{\sigma}=\left\{x \in G: \xi_{\sigma}^{x}=\xi_{\sigma}\right\}
$$

with $\left[G: S_{\sigma}\right]=t_{\sigma}$. Also for each $\sigma \in E$ we have $\sigma \cong \tau_{\sigma}^{f}$ where $\tau_{\sigma}$ is an irreducible representation of $S_{\sigma}$ satisfying $\left.\tau_{\sigma}\right|_{A_{\sigma}}=e_{\sigma} \cdot \xi_{\sigma}$. Since $E$ is local $A_{p}$ for every $\left.p \in\right] 1, \infty$ [, we have by Lemma 4 that

$$
B=\left\{\sup t_{\sigma}: \sigma \in E\right\}<\infty .
$$

Also, since $\xi_{\sigma}$ is 1-dimensional, we have for all $x \in A_{\sigma}$ that

$$
\left|\chi_{\tau_{\sigma}}(x)\right|=e_{\sigma} \cdot\left|\xi_{\sigma}(x)\right|=e_{\sigma}=d_{\tau_{\sigma}} .
$$

Thus, applying Lemma 2, we get for $p \in] 1, \infty[$ that

$$
\left\|\chi_{\tau_{\sigma}}\right\|_{p} \geqq d_{\tau_{\sigma}} /\left[S_{\sigma}: A_{\sigma}\right]^{1 / p} \text {. }
$$

Now define a continuous function $f_{o}$ on $G$ by setting 


$$
f_{\sigma}(x)= \begin{cases}t_{\sigma}^{1 / 2} \chi_{\tau_{\sigma}}(x) & \text { for } x \in S_{\sigma} \\ 0 & \text { for } x \in G-S_{\sigma} .\end{cases}
$$

Arguing precisely as in the proof of Lemma 4 we have that $f_{o} \in$ $S_{E}(G)$ and, by Lemma 3 , we have for $p \in[2, \infty[$ that

$$
\left\|f_{\sigma}\right\|_{p}=t_{\sigma}^{12-1 / p}\left\|\chi_{\tau_{\sigma}}\right\|_{p} \geqq\left\|\chi_{\tau_{\sigma}}\right\|_{p} \text {. }
$$

In particular, we have

$$
\left\|f_{\sigma}\right\|_{2}=\left\|\chi_{\tau_{\sigma}}\right\|_{2}=1 \text {. }
$$

Taking $p=4 d_{\sigma}^{2} / \log d_{o}$ and observing that

$$
d_{\sigma}=t_{\sigma} d_{\tau_{\sigma}} \leqq B \cdot d_{\tau_{\sigma}}
$$

we have from (1) and (2) that

$$
\begin{aligned}
\left\|f_{\sigma}\right\|_{4 d_{\sigma^{\prime} / \log d_{\sigma}}^{2}} & \geqq d_{\tau_{\sigma}} /\left[S_{\sigma}: A_{\sigma}\right]^{\log d_{\sigma^{\prime}} / 4 d_{\sigma}^{2}} \\
& \geqq B^{-1} d_{\sigma} /\left[G: A_{\sigma}\right]^{\log d_{\sigma^{\prime}} / 4 d_{\sigma}^{2}} \\
& \geqq d_{\sigma} / 6 B .
\end{aligned}
$$

Now, since $E$ is local $\Lambda$, there is a constant $\kappa$ such that for each $\sigma \in E$ and all $p \in] 2, \infty[$ we have

$$
\left\|f_{\sigma}\right\|_{p} \leqq \kappa p^{1 / 2}\left\|f_{\sigma}\right\|_{2}=\kappa p^{1 / 2} .
$$

Again taking $p=4 d_{\sigma}^{2} / \log d_{\sigma}$, we then see that

$$
d_{\sigma} / 6 B \leqq \kappa\left(4 d_{\sigma}^{2} / \log d_{\sigma}\right)^{1 / 2}
$$

and so we have

$$
\log d_{\sigma} \leqq 144 B^{2} \kappa^{2} \text { for all } \sigma \in E .
$$

It follows that

$$
\sup \left\{d_{\sigma}: \sigma \in E\right\}<\infty \text {. }
$$

Corollary. Let $G$ be a profinite group. The following statements are equivalent:

(i) $G$ is tall;

(ii) $\hat{G}$ contains no infinite local $\Lambda$ sets;

(iii) $\hat{G}$ contains no infinite local Sidon sets;

(iv) $\hat{G}$ contains no infinite Sidon sets.

Proof. The implication (i) $\Rightarrow$ (ii) follows immediately from the theorem while the implications (ii) $\Rightarrow$ (iii) and (iii) $\Rightarrow$ (iv) are well known (see $\S 37$ of [4]). Finally, the implication (iv) $\Rightarrow$ (i) is con- 
tained in Corollary 2.5 of [6].

Complements. A result similar to ours for compact Lie groups may be found in Cecchini [1]. An immediate consequence of our theorem is that if the dual $\hat{G}$ of a profinite group $G$ is a local $A$ set then the degrees of the elements of $\hat{G}$ must be bounded. Parker [11] has proved the same conclusion under the weaker assumption that $\hat{G}$ is a local central $\Lambda_{4}$ set. If we restrict $G$ to be a pro-nilpotent group (i.e., a projective limit of finite nilpotent groups) then a good deal more can be said with the aid of the following lemma.

Lemma. Let $G$ be a finite nilpotent group and let $\sigma \in \hat{G}$. Then we have

$$
\left\|\chi_{\sigma}\right\|_{4}^{4}>\log d_{\sigma}
$$

Proof. We show by induction on $d_{\sigma}$ that the tensor product representation $\sigma \otimes \sigma$ splits into more than $\log d_{\sigma}$ irreducible components (not necessarily pairwise inequivalent). The assertion of the lemma then follows immediately. The lemma clearly holds when $d_{\sigma}=1$. Now suppose that $d_{\sigma}>1$. By Corollary 15.6 of [3] there is a 1-dimensional representation $\rho$ of a subgroup $H$ of $G$ such that $\sigma \cong \rho^{G}$. Let $M$ be a maximal subgroup of $G$ containing $H$. Then $M$ is normal in $G$ with prime index $q$ and $\tau=\rho^{M}$ is an irreducible representation of $M$ satisfying $\sigma \cong \tau^{G}$. Let $\left\{x_{1}=1, x_{2}, \cdots, x_{q}\right\}$ be a set of coset representations for $M$. By Mackey's tensor product theorem (see Theorem 44.3 of [2]) we have

$$
\begin{aligned}
\sigma \otimes \sigma & \cong \tau^{G} \otimes \tau^{G} \\
& \cong(\tau \otimes \tau)^{G} \oplus\left[\bigoplus_{i=2}^{q}\left(\tau^{x_{i}} \otimes \tau\right)^{G}\right] .
\end{aligned}
$$

By induction $\tau \otimes \tau$, and therefore $(\tau \otimes \tau)^{G}$, splits into more than $\log d_{\tau}$ components. Thus, if $m$ is the number of irreducible components of $\sigma \otimes \sigma$ counted according to multiplicity, then

$$
\begin{aligned}
m & >\log d_{\tau}+q-1 \\
& >\log d_{\tau}+\log q \\
& =\log d_{\sigma} .
\end{aligned}
$$

Proposition. Let $G$ be a pro-nilpotent group and let $E \subset \hat{G}$ be either a local central $\Lambda_{4}$ set for a local $\Lambda_{p}$ set or some $\left.p \in\right] 1, \infty[$. Then we have

$$
\sup \left\{d_{\sigma}: \sigma \in E\right\}<\infty
$$


Proof. By our opening remarks every continuous irreducible representation of $G$ is essentially a representation of a finite nilpotent quotient of $G$. Thus, if $E$ is a local central $\Lambda_{4}$ set, then the preceding lemma shows that $\sup \left\{d_{\sigma}: \sigma \in E\right\}$ is finite. If $E$ is a local $\Lambda_{p}$ set then, since each $\sigma \in \widehat{G}$ is induced from a 1 -dimensional representation of an open subgroup of index $d_{\sigma}$, Lemma 4 shows that $\sup \left\{d_{o}: \sigma \in E\right\}$ is finite.

ExAmple. Let $G=\prod_{n=6}^{\infty} A_{n}$ where for each $n A_{n}$ is the alternating group on $n$ letters. By Theorem 2.5 of [7] $G$ is tall so $\hat{G}$ contains no infinite local $\Lambda$ sets by our theorem. However $\hat{G}$ does contain an infinite local central $\Lambda_{4}$ set. For each $A_{n}$ has an irreducible representation $\sigma_{n}$ of degree $n-1$ obtained by restricting to $A_{n}$ the irreducible representation of $S_{n}$ (the symmetric group on $n$ letters) afforded by the partition $[n-1,1]$ of $n$. From p. 766 of [9] we have that $\sigma_{n} \otimes \sigma_{n}$ splits into 4 irreducible components. Thus, if $\pi_{n}$ is the projection of $G$ onto $A_{n}$, then $E=\left\{\sigma_{n} \circ \pi_{n}: n=6,7, \cdots\right\}$ is an infinite local central $\Lambda_{4}$ set for $G$. In addition, Corollary 4.2 of [10] shows that $E$ is a central Sidon set. Thus $G$ is a profinite group which admits infinite central Sidon sets but no infinite Sidon set. In view of Theorem 9 of [13] and $\S \S 3,4$ of [6] it is unlikely that such examples exist when $G$ is connected.

The results of this paper appear in [8]. The author is indebted to his supervisor Dr. J. R. McMullen for his many suggestions and encouragement.

\section{REFERENCES}

1. C. Cechini, Lacunary Fourier series on compact Lie groups, J. Functional Analysis, 11 (1972), 191-203.

2. C. W. Curtis and I. Reiner, Representation theory of finite groups and associative algebras, Pure and Applied Mathematics XI, Interscience, New York, 1962.

3. L. Dornhoff, Group Representation Theory, Part A, Pure and Applied Mathematics 7, Marcel Dekker, New York, 1971.

4. E. Hewitt and K. A. Ross, Abstract Harmonic Analysis, I and II, Springer-Verlag, Berlin, 1963 and 1970.

5. E. Hewitt and K. Stromberg, Real and Abstract Analysis, Springer-Verlag, Berlin, 1965.

6. M. F. Hutchinson, Non-tall compact groups admit infinite Sidon sets, J. Austral. Math. Soc. Ser. A, 23 (1977), 467-475.

7. - Tall profinite groups, Bull. Austral. Math. Soc., 18 (1978), 421-428.

8. - Lacunary sets for connected and totally disconnected compact groups, Ph. D. thesis, University of Sydney, Sydney, 1977. See also: Abstract, Bull. Austral. Math. Soc., 18 (1978), 149-151.

9. F. D. Murnaghan, The analysis of the Kronecker product of irreducible representations of the symmetric group, J. Amer. Math. Soc., 60 (1938), 761-784.

10. W. A. Parker, Central Sidon and central $A_{p}$ sets, J. Austral. Math. Soc., 14 (1972), $62-74$. 
11. W. A. Parker, Local central $\Lambda(p)$ dual objects, Canad. Math. Bull., 20 (4) (1977), 515.

12. J. F. Price, Local Sidon sets and uniform convergence of Fourier series, Israel J. Math., 17 (1974), 169-175.

13. D. Rider, Central lacunary sets, Monatsh. Math., 76 (1972), 328-338.

14. G. de B. Robinson, Representation Theory of the Symmetric Group, University of Toronto Press, 1961.

15. S. S. Shatz, Profinite Groups, Arithmetic and Geometry, Annals of Mathematics Studies 67, Princeton University Press, 1972.

Received August 8, 1978.

The UNIVERSITy OF SyDNey

SYdneY, N.S.W. 2006, Australia 


\section{PACIFIC JOURNAL OF MATHEMATICS}

\section{EDITORS}

DONALD BABBITT (Managing Editor)

University of Galifornia

Los Angeles, California 90024

Hugo RossI

University of Utah

Salt Lake City, UT 84112

C. C. MoOre AND ANDrew OGG

University of California

Berkeley, CA 94720

\section{J. DugundjI}

Department of Mathematics University of Southern California

Los Angeles, California 90007

R. Finn and J. Milgram

Stanford University

Stanford, California 94305

ASSOCIATE EDITORS

E. F. Beckenbachi

B. H. NeumanN

F. WOLF

K. YOSHIDA

\section{SUPPORTING INSTITUTIONS}

UNIVERSITY OF BRITISH COLUMBIA

CALIFORNIA INSTITUTE OF TECHNOLOGY

UNIVERSITY OF CALIFORNIA

MONTANA STATE UNIVERSITY

UNIVERSITY OF NEVADA, RENO

NEW MEXICO STATE UNIVERSITY

OREGON STATE UNIVERSITY

UNIVERSITY OF OREGON
UNIVERSITY OF SOUTHERN CALIFONIA

STANFORD UNIVERSITY

UNIVERSITY OF HAWAII

UNIVERSITY OF TOKYO

UNIVERSITY OF UTAH

WASHINGTON STATE UNIVERSITY

UNIVERSITY OF WASHINGTON

Printed in Japan by International Academic Printing Co., Ltd., Tokyo, Japan 


\section{Pacific Journal of Mathematics \\ Vol. 89 , No. 1 \\ May, 1980}

David Bressoud, A note on gap-frequency partitions ................ 1

John David Brillhart, A double inversion formula ................. 7

Frank Richard Deutsch, Günther Nürnberger and Ivan Singer, Weak

Chebyshev subspaces and alternation .......................

Edward Richard Fadell, The relationship between Ljusternik-Schnirelman

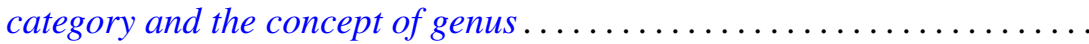

Harriet Jane Fell, On the zeros of convex combinations of polynomials.......

John Albert Fridy, An addendum to: "Tauberian theorems via block

dominated matrices" ..................................

Andrzej Granas, Ronald Bernard Guenther and John Walter Lee, Applications of topological transversality to differential equations. I. Some nonlinear

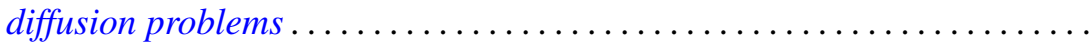

David E. Handelman and G. Renault, Actions of finite groups on self-injective

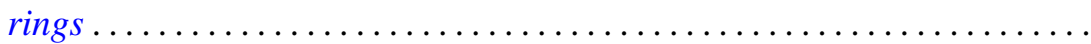

Michael Frank Hutchinson, Local $\Lambda$ sets for profinite groups .............

Arnold Samuel Kas, On the handlebody decomposition associated to a

Lefschetz fibration...

Hans Keller, On the lattice of all closed subspaces of a Hermitian space.....

P. S. Kenderov, Dense strong continuity of pointwise continuous

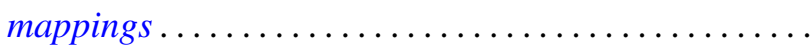

Robert Edward Kennedy, Krull rings.................

Jean Ann Larson, Richard Joseph Laver and George Frank McNulty,

Square-free and cube-free colorings of the ordinals ...

Viktor Losert and Harald Rindler, Cyclic vectors for $L^{p}(G)$

John Rowlay Martin and Edward D. Tymchatyn, Fixed point sets of

1-dimensional Peano continua...

Augusto Nobile, On equisingular families of isolated singularities ...

Kenneth Joseph Prevot, Imbedding smooth involutions in trivial bundles ...

Thomas Munro Price, Spanning surfaces for projective planes in four space.

Dave Riffelmacher, Sweedler's two-cocycles and Hochschild cohomology....

Niels Schwartz, Archimedean lattice-ordered fields that are algebraic over

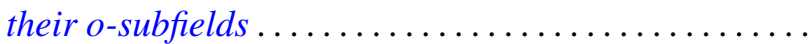

Chao-Liang Shen, A note on the automorphism groups of simple dimension groups.

Kenneth Barry Stolarsky, Mapping properties, growth, and uniqueness of

Vieta (infinite cosine) products ...

Warren James Wong, Maps on simple algebras preserving zero products. I.

The associative case ............................. 